\title{
O papel da família e das redes primárias na reestruturação das políticas sociais
}

\author{
The role of family and primary net work \\ in the reform of social policies
}

Ma u ro Serapioni 1

\footnotetext{
1 Un iversid ade Estadual do Ceará, Me s trado em Saú de Pública. Av. Pa ra n jana 1.700 Serrinha, 60740-020, Fortaleza CE.
} mauro_serapioni@yahoo.es
Abstract The crisis of Welfare State has been contributing to rediscover the family, primary networks and communities as fundamental acto rs to perfo rm so cial pol icies. The family, particularly, has attracted more and more atten tion of governmental institu tions and social sci en tists for its role of protection, help and care. Actually, there a re many proposals of social pol $i$ cies based on the conception of "community care", which aim to $h$ old the community co-responsible for so cial and health problems. The Brazilian Family Health Program is one of these strategies, whose the main objective is to provide basic health care to families and communities. However, constant changes at the organization of the family, basically in its composition, structu re and function, makedifficult development of effective policies focused on the family participation. In the light of this context, it is impo rtant to design adequate stra tegies to promote a continuing education process for health professionals, which could complement their knowledge in familiar and community approaches. Thus, social policy makers have opportunity to introduce innova tive and creative ideas at community level, which could valorize the role of informal care, specially, that del ivered by rel atives, in ord er to integra te these activi ties to them carried out by the institutional care providers.

Key words Family, Family health program, Informal care, Community care, Social policies
Resumo A crise do Estado de Bem-Estar Social tem contribuído pa ra a redescoberta da família, das redes primárias e da co munidade como ato res fundamentais na efetivação das politicas sociais. A família é cada vez mais objeto de atenção das instituições governamentais e dos ci en tistas sociais pela grande quantidade de atividades de proteção, ajuda e cuidado que ela desenvolve. Atualmente, há várias propostas de políticas sociais baseadas na concepção de "cuidado co munitário", que objetivam co-responsabilizar a co munidade em relação aos problemas so ciais e de saúde. Uma das estratégias é o Programa de Saúde da Família, que visa ofere cer serviços de atenção básica às famílias e às comunidades. Observa-se, porém, uma profunda tran sformação na organização da família, na sua co mposição e estru tu rae sua função. $O$ desenvolvi men to de uma poli tica mais efetiva nessa á rea deve promover um proce s so de educação conti nuada dos profissionais, a profundando sua formação quanto à abord a gem familiar e comunitária. Os planejado res de politicas sociais dispõem de várias possibilidades pa ra introduzir novase criativas inici a tivas em nivel de co mu nidade, que ofere cem a opo rtunidade de valorizar o papel do cuidado informal, em particular o cuidado subministrado pelo parentesco, e pa ra integrá-lo às a tividades realizadas pelos serviços institucionais. Palavras-ch ave Família, Saúde da família, Cuidado informal, Cuidado comunitário, Políticas sociais 


\section{Introdução}

A família desem penha um papel import an te na provisão de cuidado informal para seus membros. Há um geral reconhecimen to, hoje em dia, de que ela está no centro das funções de cuidadb. Uma grande parte do cuidado acon tece no lar. A vida qu o tidiana doméstica é caracterizada pelo aten dimen to às necessid ades físicas e psicológicas dos diferen tes membros da família. É no seu con texto social que se salvaguarda a saúde e se lida com as doenças. A família representa, na verd ade, a unidade básica de atenção à saúde; é o primei ro nível de atenção à saúde. Nesse senti do, o cuidado familiar con s ti tui o fundamen to do cuidado comunitário. Uma pesquisa realizada em 1996 pela $\mathrm{Bri-}$ tish Medical Association evidenciou que 80\% das doenças men ores são diagnosticadas e trat adas em casa, s em a intervenção dos profissionais de saúde (Siza, 2000). Há quase vinte e cinco anos, Levin (1979) tinha ch egado às mesmas condusões, regis tran do que $75 \%$ de todo o cuid ado com a saúde acon teciam no con tex to informal da família extensa. Ainda no Reino Unido, estima-se que cerca de 5,7 milhões de pessoas estão regularm en te envo lvidas na provisão de cuidados na comunidade (Cl a rke, 2001). Calculando o número de horas dedicadas ao cui$\mathrm{d}$ ado de familiares, alguns investigadores chegaram a estimar os gastos financei ros equ ivalen tes ao total do orçamen to dos serviços sociais e de saúde (Finch, 1989).

No geral, as pesquisas dos últimos 25 anos têm destacado o papel fundamental da família e das redes sociais na atenção e na promoção da saúde, nas três seguintes direções (Siza, 2000): 1) no fortalecimen tode relações que produ zem saúde ou increm entam a capacidade de en frentar even tos críticos e mobilizar recursos adequados; 2) no desenvolvimento da capacidade de manutenção e promoção de relações de su porte social no âmbi to do paren te sco extenso, da vizinhança e do associacionismo de ajuda mútua; 3 ) no melhoramento do acesso aos serviços de saúde, graças a sua função de mediação e de conhecimento das oportunidades e dos critérios de acesso.

As pesquisas epidemiológicas e psicossociais têm demonstrado os efei tos positivos das relações sociais na evolução de inúmeras patologias. Coesão e solidez dos laços familiares podem reduzir a percepção da gravidade dos eventos mórbidos. Segundo vários autores, a mortalidade e a morbidade são significativa- men te mais elevadas entre as pessoas com escassas relações sociais (Glenn, 1975; Cobb, 1976; Berkam \& Syme, 1979; Find, 1989; Andrade \& Vaitsman, 2002). Portanto, hoje em dia, todos recon hecem que a família con tribui, de maneira fundamental, para o bem-estar da população e recomendam que as políticas sociais, os governos e as or ganizações não-governamentais apóiem as famílias no de senvolvimen to dessa importan te tarefa.

Entret a n to, a crise do Estado de Bem-estar Social vem prom oven do, nos países industrializados, uma série de questionamen tos sobre o papel do Estado e da sociedade na resolução dos probl emas sociais (Smith \& In gram, 1993). É como se a teia de solidariedade social que embasou a criação do pró prio Estado de BemEstar Social se tivesse esgarçado, à medida que a sociedade transferiu ao Estado toda e qualquer responsabilidade sobre as demandas sociais. Nos últimos anos, há uma cla raten dência de redescoberta dos setores informais, das famílias e da comunidade como atores importantes na efetivação dessas políticas. Em outras palavras, começa-se a recuperar aqu eles sujeitos (famílias, comunidades de vizinhos, voluntariado, grupos de auto-ajuda, organizações não-govern a m entais) que, de diferen tes formas e com diferen tes níveis de envolvimen to, desenvo lvem funções assistenciais e de cuidado.

$\mathrm{O}$ atual deb a te sobre o re s su r gi m en to da família no cenário das políticas sociais é caracterizado por controvérsias e polêmicas entre as diferentes forças políticas. Tradicionalmente, as forças políticas que sustentaram a modernização haviam identificado a família como um obstáculo à modernização da socied ade. Su ce ssivamente, os liberais descobri ram as potencialidades da família em substituir algumas intervenções do Estado, que eram consideradas burocráticas e muito custosas. A partir dos anos 70 , também as forças de esqu erda começaram a recon h ecer a importância social das famílias.

An tes de analisar as políticas de saúde baseadas no cuidado familiar e comu nitá rio e antes de poder apreciar o efei to dessas políticas, é necessário compreender a natu reza das mudanças acon tecidas na estrutu ra e na organização da vida familiar na socied ade modern a. Precisamente, temos de saber como as recen tes mudanças nos modelos familiares têm afetado as habilidades das famílias para desempenhar as funções de cuidado informal. Os responsáveis pelas políticas de saúde devem estar conscientes das mudanças ocorridas nas últimas déca- 
das na vida familiar, an tes de introduzir qualqu er tipo de política pública que pretenda incen tivar o cuidado inform al, s eja da família, seja das redes de suporte social.

An tes de discutir, no en tanto, o impacto das mudanças nos modelos organizacionais da família, é preciso definir com mais cla reza o que nós en ten demos pelo termo "família".

\section{Definição do con cei to de família}

A família forma a unidade básica da organização social na nossa socied ade. Para alguém ela repres enta o pré-requisitode um sistema social estável. Muitos cien tistas sociais, en tretanto, percebem um declínio da família como instituição social e sustentam que a família tem perdido sua autoridade moral e seu sentido de responsabilidade e, somen te voltandoaos valores da família tradicional, é possível deter o declínio moral. Esse ti po de análise contém uma imagem idealizada da família: um casal heterossexual casado, incluindo os parentes biológicos da criança, formado por um único domicílio monofamiliar. Essa tipologia con sti tui o que mu itos defin em como núcleo familiar normal ou família tradicional. (Clarke, 2001)

Analisando, con tu do, dados referen tes à tipologia e estrutura da família de qualquer país ocidental, industrializado ou em desenvolvimento, pode-se observar que a família não é uma entidade fixa, mas uma pluralidade de formas. Então, diante da existência dos diferen tes ti pos de família, o uso do termo "família" pode confundir. Vários estudiosos, por exemplo, para evitar essa con trové rsia conceitual, têm preferido adotar uma abordagem mais pragmática e preferem falar de "famílias" (Clarke, 2001), de "vida familiar" (Don ati \& Di Nicola, 1996) ou, ainda, de "formas empíricas de família" (Vaitsman, 1999).

Queremos manter o termo "família" para enten der um modelo ideal de família (no sen tido jurídico-legal), ou é melh or adotar uma diferenciação social e cultu ral do termo? Em outras palavras, o conceito de família serve para designar somen te as famílias baseadas no matrimônio, ou tem de incluir, também, todas as o utras formas de família empiricamente existentes?

Não cabe ao cien tista social re s pon der a esse dilema de natu reza ética, política e jurídica. Ao cientista social cabe analisar as várias formas em que se verificam as diversas condições familiares e as implicações que cada uma tem no seu rel acion a men to com as políticas sociais.

In dependen temen te da discussão terminológica e con ceitual, a família tem de ser compreendida como: a) intercâ m bio simbólico entre gêneros e gerações; b) mediação en tre cultu ra e natureza; c) mediação en tre esfera privada e esfera pública. Nesse sentido, a família deve ser en tendida seja como relação intersubjetiva do mundo da vida, seja como instituição (Don a ti \& Di Nicola, 1996).

\section{Mudança da família}

Nas últimas décadas, houve significativas mudanças seja na estrutura e funções da família, seja na dinâmica interna da vida familiar. Essas mudanças, porém, têm implicações, também, na provisão de cuidado informal.

Do ponto de vista das funções, a família perde a sua estrutura multifuncional (unidade de produção e con sumo, deten tora de mecanismos de transmissão cultu ral de valores e normas, de integração social de seus membros, de socialização primária e secundária das novas gerações, de con trole da propried ade) que, tradicionalmente, a s sumia e que, a gora, é assumida por outras agências, como a escola, a fábrica, o mercado, os meios de comunicação, a igreja, etc. (Donati \& De Nicola, 1996). Na sociedade moderna, a família tende a se reduzir sempre mais à família nu clear, manten do um número limitado de funções, en tre as quais: a estabilização do equilíbrio da personalidade adulta e a socialização primária dos novos nascidos (Pa rs ons \& Bales, 1974). Trata-se, porém, de duas funções, que, embora residuais, são muito importantes, já que somente a família pode desempenhar. Por isso, ainda se considera importante o papel da família na sociedade moderna e con temporânea. De fato, afirmam Donati \& De Nicola (1996) que nuclearização não é sinônimo de perda de cen tralidade da família.

Do pon to de vista da estrutura, ela se transforma de extensa para nuclear. Geralmente, atri bui-se ao processo de industrialização e urbanização a responsabilidade pelo declínio da família extensa e o surgimento da unidade familiar nuclear. Essa hipótese, entretanto, tem sido questionada por vários estudiosos por várias razões:

1) considera n do o índice de ex pecta tiva de vida prevalecente nos séculos 18 e 19, não era 
muito comum a coexistência de três gerações no mesmo lar, e não era comum que os avós pudessemcuidar das crianças;

2) evidências demonstram que a industrialização não tem levado à redução do tamanho da família, mas tem incen tivado a formação de famílias extensas, uma vez que a ocupação de homens e mulheres na indústria tornou necessária a pre sença de outros paren tes nodomicílio; 3) outros estudiosos têm questionado a posição da família nuclear con temporânea como isolada do paren tesco. É questionado, em particular, o fato de a nu cl e a rização ser interpret ada como isolamen to e qu ebra das relações com o parentesco. Litwak (1960), por exemplo, contrapõe o con cei to de família nu clear de Parsons (1974) ao con cei to de "família ex tensa modificada”. Para Litwak (1960), a família isolada mantém importantes relações com a família de origem, embora não haja coabitação. Em outras palavras, a família nu clear é indepen dente como domicílio, mas conti nua sen do parte do grupo mais amplo do paren tesco. Nesse sentido, Rosenmayr e Kockeis (1963, apud Finch, 1989) falam de "intimidade a distância" para expressar essa nova con $\mathrm{fi}$ g u ração familiar. A relação extensa do parentesco e o suporte recíproco repres entam aspectos importantes da vida da família con temporânea (Clarke, 2001). Existe uma rede de paren te sco não-re sidencial que provê ajuda e suporte em caso de necessidade. Os avós e outros parentes, como demonstram muitas pesquisas, con tinuam sen do provedores de cuidado den tro da família extensa. Por isso, os sociólogos preferem falar de "família ex tensa modificada", ao invés de "família nu clear isolad a" (Morgan, 1975).

No geral, ex i s tem ainda diversas interpret ações das conseqüências dessa nova dinâmica familiar: alguns autores su stentam que o resultado dos novos modelos familiares é a progre ssiva desmembração e dissolução dos laços de parentesco e de reciprocidade familiar, até ch egar ao "final da família". Outros autores, ao con trário, analisan doos estudos empíricos re alizados sobre a família, afirmam que os signos que revelam a vitalidade das redes familiares são diversos e abundantes (Finch, 1989; Comas D’Argemir, 1997; Donati \& Di Nicola, 1996). De fato, a diversificação e a mudança das formas de convivência não significam a perda da importância da família na sociedade moderna. Segundo Comas D'Argemir (1997), a pesar das formas de convivência terem mudado profundamente, os vínculos de parentesco, especial- men te aqu eles intergeracionais, têm se reforçado, con trad i zen do o modelo pars oniano da família nuclear.

Nas últimas décadas, a família tem passado por várias crises nas sociedades industrializadas ou naqu elas em de senvo lvimento. As diferen tes crises que têm afetado a família propiciam o surgimento de novo tipo de família (e não a sua extinção, como teorizaram as corren tes evo luci onistas) e novas refl exões sobre a família. Por isso, o interesse de cien tistas sociais, de profissionais e de políticos tem sido sempre maior. Nesse sen ti do, Ferrari \& Kaloustian (1998) afirmam que a família brasileira, em meio a discussões sobre a sua desagregação ou enfraquecimento, está presente e permanece enquanto espaço privilegiado de socialização, de prática de tolerância... e de lugar inicial pa ra o exercício da cidadania.

\section{Novos padrões de família}

Entre os fatores que têm resultado no surgimento de novas formas familiares cabe destacar as mudanças na legislação que regula as relações con jugais e de filiação; a ruptura da dico tomia entre papéis públicos e privados, segundo o gênero; o crescen te processo de indivi dualização econômico e cultural dos sujeitos; a pluralização dos estilos de vida; a igualdade en tre os sexos e a con seqü en te ruptura de casamentos sentidos como insatisfatórios (Vaitsman, 1999; Don a ti \& Di Nicola, 1996).

A mudança dos modelos familiares, um dado inegável, é objeto de análise e debate nos principais fóruns internacionais. Realmente, a redução do número de filhos, o ret a rdo do matrimônio, $\mathrm{o}$ atraso da maternidade, $\mathrm{o}$ aumen to da porcentagem de divórcios e o incremen to das uniões de fato representam alguns dos indic adores familiares que caracterizam os países industrializados, como também mui tos países em de senvolvimen to. Esses fenômenos têm tido um impacto rel evan te na estrutura dos núcl eos familiares, produzin do significativas mudanças e novas formas de or ganização familiar (De Ussel, 1996). Ju n to com o padrão de família nu clear que ainda representa o arranjo residencial predominante, ob serva-se o surgimento das seguintes tendências de organização da família: 1) pluralização das formas familiares; 2) aumento das famílias monoparentais; 3 ) aumen to das famílias com um único el emento; 4) surgimento das famílias reconstituídas. 
Também no Brasil, segundo Vaitsman (1999), ob s erva-se esse mesmo processo de diferen ciação da estrutura familiar: O Brasil jamais foi uma sociedade homogênea quanto às suas formas de família... Atualmente as formas empiricas de famílias correspondem cada vez menos à família conjugal moderna estável e nuclear. Este mod elo en trou em cri se. Uma pesquisa realizada em Florianópolis em 1993 (Rech et al., apud Takashima, 1998), con st a tou que $73,3 \%$ das famílias entrevistadas eram de ti po nudear; dessas, $40 \%$ se autodenominavam "desestruturadas", ou seja, "com problemas na dinâmica de in teração”. As famílias matrifocais representavam $23,3 \%$ da amostra. O dado surpreen den te dessa pesquisa - na opinião de Takashima (1998) - foi o fato de que 64\% dos "pais físicos" eram considerados ausentes pelas mães en trevistadas. Os mais recen tes dados do censo dem ográfico sobre População e Domicílios do IBGE (IBGE, 2000) confirmam essa tendência. A proporção de mulheres responsáveis pelo domicílio passou de 18,1\%, no ano 1991 para 24,9\% no ano 2000.

\section{Família, funções assistenciais e políticas sociais}

Há um con s enso entre os cientistas sociais em reconhecer uma correlação inversa en tre funções exercidas pelas famílias e de senvo lvim en to de políticas sociais. Historicamente, o aumen to de serviços ofert ados pelo Estado de Bem-Estar corresponde a uma contração das funções familiares, ou seja, qua n to mais funções são concentradas nas famílias, tan to menos rel evante é o papel das agências de serviços extrafamiliares e vice-versa (Donati \& Di Nicola, 1996). Essa correlação, con tu do, tem duas diferen tes interpretações: a) uma interpretação negativa por parte de quem enfatiza os efeitos de erosão e debilitação cultu ral e social da família, como conseqüência da contínua intrusão dos mecanismos de políticas sociais e de outras agências de mercado, efei tos que Habermas (1997) chama de colonização do mundo da vida e que reduzem a família a uma simples esfera privada e dependente das instituições do Estado de Bem-Estar. A con s eqüência mais marcan teé o fato de a família ser sempre mais regulada por códigos alheios que quebram a solidaried ade familiar; b) uma interpretação positiva no sentido de que a família nuclear - sempre mais reduzida no número de seus componen tes e, portanto, com menos recursos humanos para lidar com todos os probl emas rel acionados à reprodução primária - precisa de uma rede de serviços que a libertem do fardo de desempenhar inúmeras t a refas e facilitem a sua dedicação prioritária às funções afetivas (Donati \& Di Nicola, 1996). Embora sustentadas por corren tes ideológicas opostas, ambas as interpretações dessa correl ação apontam para uma mudança nas relações entre família e instituições de welfare state e uma revalorização do sistema de cuidado informal. De fato, é importante superar a lógica atualmen te prevalecen te de satisfação das necessidades, fortemen te baseada na polarização entre serviços ofertados pelas instituições públicas e serviços adquiríveis no mercado. Essa polarização se sus tenta na aceitação do pres supos to de que, na sociedade con temporânea, a família é isolada do paren te scoe de sua comunidade. Entretanto muitas pesquisas têm demonstrado com o, a pesar das mudanças ocorridas na estrutura da família e nas relações comunitárias, o campo das redes sociais e da solidariedade primária (relações de parentesco, de amizades e de vizinhos) persiste e continua tendo uma significativa rel evância para os sujeitos, também nas socied ades urbanizadas (Don a ti \& De Nicola, 1996). Realmente, a dinâmica demográfica (com redução da natalidade e o envelhecimen to da população) e as novas tendências econômicas (com efei tos no trabalho e nos índices de emprego dos jovens), junto a outros fatores socioecon ô mi cos e culturais, têm con tribuído para increm entar os intercâmbios material, social e afetivo entre diferen tes gerações (Comas D’Argemir, 1997). Da mesma forma, têm aumentado, também, as tradicionais funções assistenciais exercidas pela família, entre elas, as de proteção, de ajuda e de cuidado das pessoas dependentes por motivos de saúde ou de idade. Essas atividades são pou co visíveis porque não se fazem no marco das relações trabalhistas e são caracteri $z$ adas por sua dimensão moral e afetiva (Comas D’Argemir, 1997). Cabe destacar, de acordo com a experiência de muitos países ocidentais, que a maior parte das funções assistenciais realizadas no âmbi to da família ou em nível de comunidade é realizada por mulheres. Finch (1989) afirma que, no Reino Unido, segundo as fon tes estatísticas, a grande maioria de pessoas recebe cuidados por parte do paren te sco e que as mulh eres subministram entre 75 e $85 \%$ desses cuidados.

Inicia tivas de solidariedade e de ações coletivas de apoio representam, segundo Takashi- 
ma (1998), "formas de organização cotidianas das famílias e experiências vitais" dos setores mais necessitados da população da América Latina. Em relação ao Brasil, as pesquisas têm revelado várias atividades e redes de solidariedade no nível doméstico ou no nível comunitário. Takashima (1998), por exemplo, tem iden tificado três áreas de or ganização solidária: a) redes espontâneas de solidariedade en tre vizinhos - casos de morte, de incêndio, doença etc.; b) prá ticas info rmais organizadas - aprópria comunidade assume a criança abandonada, denuncia casos de violência etc.; c) práticas fo rmalmen te organizadas, com agen te externo motivador. Exemplos: sacolão; fábrica de sabão; projeto de geração de renda...

A val orização das redes sociais e da família é quase con temporânea ao surgimento da crise econômica e fiscal dos Estados de Bem-Estar. Nesse con tex to, sublinha Carvalho (1998), re ssurge também a família e a comunidade. Essa convergência tem, de fato, l evado a recon h ecer o papel das redes sociais e, no geral, do chamado terceiro setor como import a $\mathrm{n}$ tes atores para satisfa zer as necessidades sociais. Obviamente, essa posição pode assumir diferen tes significados:

1) Pode ser en tendida como uma resposta à crise econômica e fiscal do Estado de Bem-Estar valorizando o trabalho da família e das redes sociais primárias, o que significa, como afirmam Donati \& Di Nicola (1996), "uma reprivatização de serviços e modalidades de satisfação das necessidade s" e, paralelamente, um parcial redimensionamento do empenho do Estado em relação às necessidades das comunidades.

2) Pode ser en tendida como uma resposta às novas necessidades e demandas relacionadas mais à qualidade de vida, que à segurança material. Nesse sentido, a valorização de cuidado in formal e de redes sociais poderia representar uma maior preocupação do Estado com os aspectos rel acionais, de humanização e de pers onalização das intervenções sociais. Se isso acontecesse, estaríamos diante de um processo de transição que nos leva ria em direção a uma sociedade de serviços, ou seja, estaríamos diante de uma en orme mudança socioeconômica e cultural: a passagem de um Estado de Bem-Estar a uma Sociedade de Bem-Estar (Donati \& De Nicola, 1996).

No con tex to do Brasil, a retom ada da família e das redes sociais como referência das políticas públicas éjustificada, também, como a estratégia mais adequada - ao lado das intervenções sociais tradicionais (saúde, educação, ha- bitação, renda, etc.) - para de senvo lver programas sociais efetivos para enfrentar a pobreza (Draibe, 1998).

\section{Família e políticas de saúde}

A necessidade de uma mudança na relação família-políticas sociais tem marcado o debate in ternacional e nacional nos últimos 15 anos. Rel a tiva men te ao Brasil, vários estudiosos vêm criticando o trabalho até hoje desenvolvido com as famílias. Carvalho (1998) afirma que a família tem sido uma ilustre desconhecida nas diretrizes e programas propostos pela politica social brasilei ra e recom enda retomar a família e a comunidade como pontos de partida de práticas sociais alterativas e não simplesmente alterna tivas. Isso significa que é urgente implem entar intervenções e atividades de apoio que possam alterar a qualidade da vida e a exclusão social das famílias brasileiras. Para Vascon celos (2000), as atenções prestadas à família são consideradas práticas conservadoras e po u co eficientes, "porque estão presas a uma cultura tutelar de relação com as classes populares", que não aceita a "a utonomia da família por não confiar em sua capacidade". Um exemplo destas práticas, segundo Carvalho (1998), é "a enorme resistência a programas de complementação da renda familiar já existentes... há de zenas de anos em vários países do mundo". Essa postura resulta - ressalta Vasconcelos (2000) - em aumen tos de custos dos programas, em expansão exa gerada da burocracia gestora e em perda de qualidade.

Outra crítica se refere à persistente fragmentação e individualização das políticas sociais dirigidas às famílias, que, segundo Takashima (1998), provocam "duplicidade ou descontinuidade no atendimento às famílias" e não integram os diferen tes proj etos, resultando em uma série de ações atomizadas. Recomenda-se, portanto o surgimento de propostas de integração das diferen tes intervenções no campo social, com vistas a su perar a atual fragmentação, s eja das políticas públicas (direi to à saúde, à educação, àhabitação, ao tran sporte, à segu rança etc.), seja das do indiví duo (direitos da mulher, da gestante, da criança, do idoso, do trabalhador etc.). A grande maioria das políticas sociais, de fato, se dirige - segun do Vas concelos (2000) - para o "atendimento individualizado das pessoas, descon si derando o universo familiar e comunitário em que vivem". A a ten- 
ção à família - acrescenta Carvalho (1998) tornou-se periférica. Quando exi stente, não era o alvo, mas sim a mulher, o trabalhador, a criança.

Igualmentequestion adas são as políticas de saúde volt adas para a família, con s i deradas ainda centradas em práticas tradicionais. Embora se reconheça a importância do Programa de Sa ú de da Família (PSF) como primeira estratégia de reorientação do modelo de atenção à saúde, vários estudiosos evidenciam sua abor$\mathrm{d}$ a gem ainda fundamen $\mathrm{t}$ ada no individualismo assistencial. Pa ra Neto (2000), é preciso de senvo lver, no âmbi to dos serviços de atenção primária, uma abordagem familiar que considere a família, não somente como geradora de crises, mas também de soluções.

$\mathrm{Na}$ experiência cotidiana, observa-se que qualqu er ti po de intervenção da equipe do PSF é con s i derado familiar: Nas visitas às famílias, $a$ atenção fica muito dirigida aos aspectos que os diversos programas priorizam, como a amamentação, o uso de re-hidratante oral, o controle da hipertensão, etc. (Vasconcelos, 2000). Nesse senti do, é a unidade familiar, e não os indivíduos, que deve ser tomada como base de referência para desenhar os programas dirigi dos à saúde da família (Draibe, 1998). Para Feu erwerker \& Al m eida (2000), o probl ema maior que a estratégia do PSF en frenta para sua consolidação e ampliação en con tra-se na área de recursos humanos. Não há ainda profissionais com perfil, competências e habilidades necessárias para compor uma equipe apta a desenvolver uma a bord a gem familiar. O utro probl ema premente é a dificuldade de en contrar soluções definitivas para as relações trabalhistas com os profissionais do PSF, que permitam resolver o problema da rotatividade (Bessa \& Penaforte, 2002).

De qualqu er forma, a valorização da família e das redes sociais, no con texto da crise do Welfare St a te, refl ete certa consciência do esgotamen to da opção pelo indivíduo como ei xo das políticas e dos programas sociais. Hoje em dia, em níveis internacional e nacional, há um consenso sobre a importância de 1) retomar a família como unidade de atenção das políticas públicas; 2) desenvo lver redes de apoio e de envo lvi men todas famílias e comunidades; 3 ) re alizar uma mel h or integração en tre famílias, s erviços públi cos e iniciativa do setor informal.

\section{Uma nova proposta de política social: o cuidado comunitário}

Atualmente, há um gran de número de propostas de políticas sociais baseadas na con cepção de "cuidado comunitári o" (Community Care), que aproximam a assistência institucional e a não-institucional, a formal e a informal, incluindo as redes de solidariedade primárias (parentesco, amigos, vizinhos) e o voluntariado. A proposta de Community Care surge nos anos 70, na área de saúde mental, como crítica às modalidades de provisão dos serviços institucionais: Havia o conven cimen to que qualquer forma de cuidado comunitário era preferível ao tratamen toden tro das instituições (Clarke, 2001). No Reino Unido, a Co mmunity Care foi amplamen te discutida entre cien tistas sociais e atuan tes no campo dos serviços sociais e de saúde. O mesmo Relatório Griffiths (1988) recom endou aos serviços públ i cos desem pen ha rem um forte papel de suporte a favor dos recursos de cuidado informal: famílias, amigos, vizinhos e outras pessoas locais. Sucessivamente, no ano 1990, o governo con s ervador desse país lançou uma reforma do setor saúde (National Health Service and Community Act), baseada em alguns princípios do cuidado comunitário, com o prop ósi to de reequilibrar a relação en tre cuidado formal e informal, e de estabelecer parceria en tre estado, indivíduos, famílias e grupos de voluntariado (Aarke, 2001). Vale ressaltar que o governo conservador não qu eria som ente envolver famílias e grupos de voluntariado na provisão do cuidado, em con cordância com as filosofias prof i s s i onais e os valores de desin stitucionalização e descentralização desse momen to, mas pretendia também reduzir o papel de prestadores das autoridades locais de saúde e, em con seqüência, con ter a despesa sanitária. De fato, como aponta Ba rnes (1997), o incen tivo à prestação de cuidado informal é, s em dúvida, uma política custo-efetiva, mas, ao mesmo tem po, representa uma estra té gia de privatização da Com munity Ca re.

$\mathrm{Na}$ perspectiva sociológica da política social baseada no cuidado comu n i tá rio (e não somen te na pers pectiva econômica), as famílias e as redes informais da comunidade devem ser con si deradas não apenas destinatá rias da atenção à saúde, mas também sujeitos ativos que podem con tribuir, com recu rsos pró prios, para o processo de produção da saúde. A idéia básica do "cuidado comunitário" é a co-respon sabilização e a participação da comunidade local 
em relação aos probl emas sociais e de saúde.

Entret a n to, isso não significa, na opinião de Folgheraiter (1991), que as insti tuições formais subministram cuidados den tro da comunidade (care into the community). Essa era a idéia ess encial da descentralização dos serviços na comunidade que tem caracterizado a primei ra fase do Estado de Bem-Estar Social, que incluía a assistência domiciliar e os serviços descentralizados em nível local. Essa política de desinstitucionalização dos serviços de saúde, embora tenha ofert ado serviços essenciais às famílias e comunidades, não pode ser ainda considerada uma política de co mmunity care, na medida em que não se preocupa em fortalecer a auton omia das famílias, da comu nidade e, no geral, das redes primárias. A premissa do cuidado comu nitário é que a comunidade se torne um sujeito a ut ô nomo de produção de cuidado, den tro dela mesma (care by community), cujo papel é não somente identificar problemas, mas também gerar soluções.

É importante, também, salientar que a co mmunity care supõe que ainda ex i s tem sólidas relações comunitárias. Analisando, contudo, as atividades de cuidado informal que se desenvolvem na comunidade, observa-se que a maioria delas é realizada pelas famílias e pelo parente s coe, quase sem pre, por parte da população feminina. Famílias e parentesco não são a mesma coisa que comunidade. Comunidade implica relação, participação e solidariedade entre as diversas células de um âmbi to geográfico. Para evitar a "natureza problemática" e a escassa definição do con cei to de "comunidade", alguns autores têm propos to termos alternativos. Abrams e colaboradores (1989) e Baldwin (1993), por exem plo, preferem falar de "cuidado vicinal" (neighbourhood care) para identificar aquela parte da comunidade que está envo lvida em ativid ades de ajuda e su porte em favor de pessoas que moram nas redondezas de um lugar. Bulm er (1987) tem su geri do o termo "grupo primário" (primary group), composto de vizinhos, amigos e paren te sco. Para Bulmer (1987), essa definição con si dera a proximidade geográfica, os interesses compartilhados e os laços afetivos a base da relação social e do cuidado informal. O con cei to de "gru po primário" elaborado por Bulmer (1987) torna explícito o fa to de que a família e o parentes co representam a maior fon te de cuidado comunitário.

De qualquer forma, é preciso que as intervenções sociais que visam a desenvolver atividades de cuidado informal se preocupem em reforçar as relações sociais e os laços comu nitários em nível de parentesco, de vizinhança ou de grupos primários.

\section{Algumas estratégias de cuidado comunitário}

Os planejadores de políticas sociais dispõem de múltiplas possibilidades para introduzir novas e cria tivas iniciativas em nível de comunidade, que oferecem a oportunidade de valorizar o papel do cuidado informal, em particular o cuid ado subministrado pelo paren te sco, e para in tegrá-lo às atividades realizadas pelos serviços institucionais. Nesse sen ti do, o cuidado comunitário pode oferecer uma ampla gama de respostas dentro de um continuum formal-informal de divisão do trabalho assistencial. De fato, as atividades de cuidado não-profissional - realizadas por diferen tes atores, tais como a família, as redes primárias (parentes co, vizinhos e amigos) e as redes mais organizadas ( gru po de auto-ajuda, volunta riado etc.) - podem des envolver um papel significativo em relação às novas patologias crônico - degenerativas e psicossociais, que geralmente requerem uma assistência de longo prazo.

O grande desafio, porém, é conseguir formas de entrelaçamen to, em nível local, entre assistência formal e informal, para garantir, seja um mínimo de coordenação necessária, seja o respei to e o reconhecimen to da lógica divers a e das diferentes peculiaridades de cada uma (Giarelli, 2003). Trata-se de planejar a atenção de maneira que a subministração dos serviços dos profis si onais de saúde e a assistência informal realizada pelas famílias, vizinhos e amigos possam reforçar-se reciprocamente. Esse tipo de integração é desejável em tese, mas difícil de s er realizado, sobretudo pela tendência de as or ganizações de saúde formais bu rocra tiza rem e colonizarem o setor informal (Habermas, 1997). Nesse sentido, Baldwin (1993) assinala que, tradicionalmente, os serviços de co mmunity care foram des en hados a partir de abord agens hierá rquicas e verticais, baseadas em estratégias de rac i onalização econômicas dos serviços, ao invés de serem orientadas pela filosofia dos usuários.

Apesar das dificuldades apontadas, Bulmer (1991) apre s enta algumas estratégias para fort alecer o papel do cuidado informal em nível local: a) estimular a sociabilidade entre vizinhos de casa, para increm entar as relações interpessoais 
de vizinhança. Um estudo realizado na Inglaterra tem demonstrado que as estrutu ras de relação entre vizinhos poderiam oferecer uma quantidade relevante de apoio social;

b) promover grupos de auto-ajuda em nível comunitário. Existem ex periências nesse setor (pais de crianças com distúrbios mentais, familiares de pacientes que receberam alta do hospital etc.) que demonstram que o apoio recíproco de pessoas que vivem a mesma experiência pode constituir uma forma vital de cuidado comu n itário;

c) mobil izar as redes de su po rte social para identificar as pessoas que têm necessidade de cui$\mathrm{d}$ ado e que ainda não são con $\mathrm{h}$ ecidas pelos serviços sociais;

d) descentralizar a subministração de assistência formal, em nível de bairro e domicílio, para que os profissionais dos serviços fiquem mais perto das pessoas assistidas. A experiência já realizada tem demonstrado que essa tarefa é factível, embora seja preciso trabalhar a coordenação dos diferen tes serviços;

e) reforçar a assistência formal em nivel domiciliar para as pessoas idosas e não-auto-suficientes. Trata-se de uma importante atividade, s obretu do para as pessoas que vivem longe de seus familiares;

f) dar maior suporte a quem assiste. Reconhece-se que o peso da assistência que recai nos cônjuges e, sobretudo, nas mulheres, é tão grande que se faz necessário dar su porte às pessoas que assistem a outros. Por isso, é necessário organizar "serviços de trégua" ou de internação momentânea do assistido numa instituição apropriada.

\section{Algumas con siderações con clusivas}

Ao final destetrabalho, parece-nos importante, para evitar mal-entendidos e interpretações não adequadas, sublinhar algumas premissas que têm ori en $t$ ado essas reflexões.

1. A ênfase à importância da família na reorganização das políticas sociais não significa devo lver à família o "fardo" da crise do Estado de Bem-Estar Social, chamando os sujeitos a retomar velhas e novas responsabilidades assistenciais. Essa premissa é importante para evitar que as considerações aqui elaboradas sejam confundidas com as políticas de "privatização forçadas" desejadas e/ou implementadas para as corren tes políticas conservadoras e neoliberais. Vale ressaltar que, também dentro da cul- tura progressista e de esquerda, a community care tem recebi do, pelo menos no passado, uma avaliação crítica, ten do sido considerada um conjun to de atividades assistenciais delegad as aos sujeitos (geralmenteàs mulh eres) que, tradicionalmente, eram respon sá veis pelo cuidado informal (Waerness, 1989). Mais recen temente, como destaca também Draibe (1998), "pode-se constatar uma saudável redução das polarizações das ideologias, e a família pode passar a ser vista sem tantos ranços e vieses anacrônicos...”, de forma que a mesma co $m m u$ nity care pode ser valorizada ou criticada por sua flexibilidade, sua potencialidade de reduzir a lógica bu rocrática da organização do aten dimen to e para aprimorar a relação en tre cuidado formal e cuidado informal. E não pelo simples fato de ser consi derada uma política social de cunho neoliberal.

A community care pode ser considerada uma resposta adequada à crise do Estado de Bem-Estar e da organização bu rocrática dos s erviços sociais e de saúde, de s de que:

a) não sejam desmantel adas as políticas públ icas tradicionais: a co mmunity care não deve legitimar o desmantelamen to dos serviços de saúde existentes. De fato, a co mmunity care não preten de renunciar à cultura de organização e planejamento adotada pelos modelos de or ganização bu rocráticos. Em outras palavras, quem critica radicalmen te o Estado de BemEstar Social e deseja uma sua total "desregulamentação" não tem a capacidade de imaginar as de s a strosas con s eqüências de uma socied ade privada dos serviços socioassistenciais básicos. O informal, por si mesmo, não poderia resolver os grandes problemas de nossa época (Folgheraiter, 1991);

b) haja uma pluralização dos atores do sistema assistencial e de cuidado, passando de um ator único (o estado) a múltiplos atores institucionais e não institucionais de política social (famílias, parentesco, redes de vizinhos e de amigos, voluntariado etc.);

c) haja uma requalificação das intervenções assistenciais baseadas em novas modalidades de organização e de trabalho, que iden tifiqu em as famílias e as redes informais das comunidades, não som en te como destinatárias das pre stações, mas também como su jei tos que possam con tribuir com rec u rsos próprios (às ve zes insubstituí veis) no processo de atenção à saúde .

2. A co mmunity care não é som en te uma nova ma neirade submin is trar o cuidado - de acordo com a filosofia que a resposta deve ser a mais 
próxima possível do nível em que su r ge a nece ssidade. Ela representa, também, uma legitimação social do setor informal, das redes sociais primárias, que se tornam su jeitos com tarefas e res ponsabilidades de cuidado. Nesse senti do, a commun ity care recon h ece que a) a maior parte das demandas de atenção já en contram uma re s posta no nível informal, sem envo lver serviços públicos e profissionais de saúde (Comas D’ Ar gemir, 1997); b) muitas necessidades, que têm ori gem na queda das relações pri má rias (setor informal) ou no seu mau funcionamento, não podem sempre ser satisfeitas através dos s erviços or ganizados (setor formal). Há que reconhecer que existem necessidades que podem $s$ er sati s faitas som en te por en ti d ades particularmente flexíveis e den tro de um con texto relacional (Donati e Di Nicola, 1996); c) há sempre uma exigência maior de assistência personaliza$\mathrm{da}$, relacionada a diferen tes e novos estilos de vi$\mathrm{da}$, mas atentas aos aspectos rel acionais, a fetivos e psicológicos das pessoas. Como aponta Dra ibe (1998): "É principalmente no campo dos chamados serviços sociais pessoais... in evit avel m ente atravessados por laços afetivos" que se há de recon $h$ ecer a liberd ade dos indiví duos e das fa- mílias de buscarem alternativas assistenciais. Nesse sentido, compartilhamos com Litwak (1960) a introdução do con ceito de "Funções compartilhadas", para ex pressar a idéia de um papel com plem entar en tre sistema formal e informal de cuidado. $\mathrm{O}$ autor, em outras palavras, a ponta para a necessidade de articular o setor formal com o setor inform al, con s i derados dois subsistemas distintos, porém integrados siner gicamente, paraque funcion em den tro de uma estrutu ra de rede, com recíproco reforço.

3. Finalmente, é importante desenvolver novas políticas sociais que reconheçam o valor do trabalho assistencial das mulheres e que, em vez de reduzir as intervenções públicas, realizem uma melhor integração en tre a rede de serviços públ i cos e as iniciativas de serviços informais (famílias, grupo de apoio etc.), para fins de envolver outros membros da família, em particular os hom ens no papel de cuidadores (Fadiga Zanatta, 1993). De fato, como reforça também Comas D’Argemir e Roca (1996), as novas situações de dependência física e psicológica demandam uma dedicação maior no âmbito privado, que mostra ser necessária uma maior redistribuição do trabalho entre homens e mulheres.

\section{Agradecimentos}

O autor agradece à Fundação Ce a rense de Apoio ao Des envolvimen to Científico e Tecnológico (Funcap) pela bolsa con cedida no período de maio/2004 a dezembro 2005. O autor também agradece ao profe s sor Itamar Filgueiras e ao jornalista Zach a rias Be zerr a de Ol ivei ra, pel a revisão do tex to em português, e a Hel ena Carvalhedo e João Santiago Martins, do Centro de Documentação da Escola de Saúde Pública do Ceará, pelo apoio e disponibilidade. 


\section{Referências bibliográficas}

Abrams P, Abrams S, Humphrey R \& Snaith R 1989. Neighbourhood care and so cial policy. Departm ent of Health, HMO, Londres.

Andrade GRB \& Vaitsman J 2002. Apoio social e redes: conect an do solidari ed ade e saúde. Ciência \& Saúde Coletiva 7(4):925-934.

Bal dwin S 1993. The mith of community care. Ch a pman \& Hall, Londres.

Ba rnes M 1997. Care, community and citizens. Longman, Londres.

Bessa O \& Penaforte J (orgs.) 2002. Médico de família. Formação, certificação e educação Continuada. Escola de Saúde Pública do Ceará, Fortaleza.

Berkam LE \& Syme SL 1979. Social net works, host resistance and mortality: a nine-year study of Alameda County residents. Amercian Jou rnal of Epidemiology 109.

Bulmer M 1987. The so cial basis of community care. All en \& Unwin, Londres.

Bulmer M 1991. Famiglia e community care oggi, pp. 257270. In Donati e Ma t teini (orgs.). Quale politica per quale famiglia in Europa. Angeli, Milão.

Carvalho MC 1998. A priorização da família na agenda da política social, pp. 93-108. In SMKaloustian (org.). Família brasilei ra a ba se de tudo. Unicef-Cortez, Brasília-São Paulo.

Clarke A 2001. The sociology of healthcare. Pearson Education, Londres.

Cobb S 1976. Social support as a modera tor of 1 i festress. Psychomatic Medicines 38.

Comas D’ Argemir D 1997. Els canvis en les estructu res i formes de convivència, pp. 9-36. In La nu ova Eu ropa social i els..., Diputació de Barcelona, Barcelona.

Comas D’ Ar gemir D \& Roca J 1996. El cuidado y la asistencia como ámbi to de ex presión de la tensión en tre bi o l ogía y cultura, pp. 57-69. In J Contreras (coord. ). Reciprocidad, cooperación y organización comunal, VIIo Congreso de Antropologia.

De Ussel JI 1996. Cambio recientes de la familia española. Sociedad y Utopia, Revista de Ciencias Sociales 7.

Donati P \& Di Nicola P 1996. Lineamenti di sociologia della famiglia. La Nuova Italia Scientifica, Roma.

Draibe SM 1998. Por um reforço da proteção à família: con tri buição à reforma dos programas de assistência social no Brasil, pp. 109-130. In SM Kaloustian (org.). Familia bra silei ra, a ba se de tudo. Unicef-Cortez, Brasília-São Paulo.

Fad i ga Zanatta L 1993. Donne epo li tica sociale. Una lettura al femminile dellepolitichesociali, pp. 47-68. In P Donati (org.). Fondamenti di Politica Sociale. La Nuova Italia Scientifica, Roma.
Ferra ri M \& Kaloustian SM 1998. In trodução, pp. 11-15. In SM Ka lo u stian (org). Famíliabrasilei ra, a ba se de tudo. Unicef-Cortez, Brasília-São Paulo.

Feuerwerker LCM \& Almeida M 2000. O programa de saúde da família e o direito à saúde. Olho Mágico 6(22):22-29.

Finch J 1989. Family, obligation and so cial chance. Polits, Oxford.

Folgh era i terF 1991. Quale com mu nity care? Una teoria e un modello organizzativo per l'innovazione, pp. 4772. In Fol gh era i ter e Don a ti (orgs.). Co mmunity care. Te o ria e pra tica del lavo ro sociale di rete. Cen tro Stu di Erikson, Trento.

Giarelli G 2003. Il malessere della med icina. Un confron to internazionale. Angeli, Milão.

Glenn ND 1975. The contribution of marriage to the psychological well-being of males and females. Journal of Marriage and the Family 3.

Griffiths R 1988. Community care agenda for action. H.M.S.O, Londres.

Ha bermas J 1997. Teoria dell'agi re comunicativo. Il Mulino Bolonha.

IBGE 2000. Censo demográfico 2000: Características da população e dos domicílios. Rio de Janeiro.

Levin L 1979. Self-care lay initiatives in health. Prodist, NovaYork.

Litwak E 1960. Occupa tional mobi li ty and ex ten ded family cohesion. American Sociological Review 25(1):7196.

Mor gan DH 1975. Social theory and the family. Rutledge and Kegan, Londres.

Neto MM 2000. En foque familiar na formação do prof i ssional de saúde. Olho Mágico 6(22):5-9.

Parsons T \& Bales RF 1974. Famiglia e socializzazione, Mondadori, Milão.

Siza R 2000. Famiglia, relazioni sociali e salute. Sociologia e Professione 36-37.

Smith SR \& In gram H 1993. Pu blic Policy for Democracy. The Bookings Institution, Washington.

Takashima GM 1998. O desafio dapolítica de aten di mento à família: dar vida às leis - uma questão de postura, pp. 77-91. In SM Kaloustin (or g.). Família brasileira, a base de tudo, Unicef-Cortez, Brasília-São Paulo.

Vaitsman J 1999. Dimensões sobre família e gênero no Brasil. RASPP - Revista Associação Pública Piauí 2(1): 63-70, jan-jun.

Vasconcelos EM 2000. A priorização da família nas políticas de saúde. Revista de APS 2(4):20-28.

Waerness K 1989. Assistenza formale ed informale nella terza età. Cosa c'è di sbagliato nella nu ova ideo logia dell'assistenza comunitaria nel wel fa restate scandinavo. La Ricerca sociale 38

Arti go apres en tado em 6/10/2004

Aprovado em 9/08/2005

Versão final apres en tada em 12/09/2005 